\title{
SARMAŞIK YAPILI TEK-FAZ DOĞRULTUCUNUN FARKLI AKIM KONTROL YÖNTEMLERİYLE PERFORMANS ANALİŻ
}

\author{
Fatih Karık ${ }^{1, *}$, Ires Iskender ${ }^{2}$, Ahmet Karaarslan $^{3}$, Naci Genç ${ }^{4}$ \\ ${ }^{1}$ T.C. Enerji ve Tabii Kaynaklar Bakanlığı, Yenilenebilir Enerji Gen. Müd., Ankara, Türkiye \\ ${ }^{2}$ Elektrik-Elektronik Mühendisliği Bölümü, Gazi Üniversitesi, Ankara, Türkiye \\ ${ }^{3}$ Elektrik Mühendisliği Bölümü, Afyon Kocatepe Üniversitesi, Afyon, Türkiye \\ ${ }^{4}$ Elektrik-Elektronik Mühendisliği Bölümü, Yüzüncü Yıl Üniversitesi, Van, Türkiye \\ fatihkarik@gmail.com, iresis@gazi.edu.tr, akaraarslan@gmail.com, nacigenc@yyu.edu.tr
}

(Geliş/Received: 19.04.2013; Kabul/Accepted: 23.07.2014)

\section{ÖZET}

Bu çalışmada, sürekli iletim modunda çalışan sarmaşık yapılı tek-fazlı doğrultucu için güç faktörü düzeltme kontrol yöntemlerinin detaylı analizi yapılmıştır. Sarmaşık yapılı doğrultucu devresinde oransal-integral kontrol, ortalama kayan kip kontrol ve öngörmeli akım kontrol yöntemleri kullanılarak birim güç faktörü (UPF) ve giriş akımının toplam harmonik bozulumu (THD) incelenmiştir. Sarmaşık yapılı güç faktörü düzeltici devrenin güç yoğunluğunu arttırma, giriş akım dalgacığını ve çıkış kapasitörünün etkin akımını azaltma, filtre büyüklügüunü düşürme gibi bir çok avantajı vardır. Sarmaşık yapılı dönüştürücünün $50 \mathrm{kHz}$ anahtarlama frekansı ve $1 \mathrm{~kW}$ güç değerinde benzetim ve deneysel çalışmaları gerçekleştirilmiş ve kontrol yöntemlerinin analizi yapılmıştır. Analizlerde, dönüştürücünün tüm kontrol yöntemlerinde yük değişimlerinde dayanıklı yap1 sergilediği gözlenmiştir. Ayrıca, öngörmeli kontrol yönteminde işlemlerin bobin akımı ölçülmeden yapılması diğer iki kontrol yöntemine göre daha avantajlı olduğunu göstermiştir.

Anahtar Kelimeler: Sarmaşık dönüştürücü, PI kontrol, Öngörmeli akım kontrol, Ortalama kayan kip kontrol

\section{PERFORMANCE ANALYSIS OF INTERLEAVED SINGLE-PHASE RECTIFIER USING DIFFERENT CURRENT CONTROL METHODS}

\begin{abstract}
In this study, a comprehensive performance analysis of power factor correction control methods are presented for an interleaved single-phase rectifier operating under continuous conduction mode. The control of interleaved converter is achieved using proportional-integral control, average sliding control and predictive current control methods to obtain unity power factor and lower total harmonic distortion of input current. The interleaved power factor correction converters have many advantages in increasing power density, reducing input current ripple and RMS current of the boost capacitor, reducing filter volume, and cutting down the cost of materials. The analysis of the interleaved converter is presented and verified exactly by simulations and experimental carried out on $1 \mathrm{~kW}$ power and $50 \mathrm{kHz}$ switching frequency. The performance of the current control methods for the interleaved converter is investigated by Matlab/Simulink program for different cases of operation such as change in input voltage and load. It is observed from the analysis that predictive current control method is more suitable than the others because it doesn't need sense current.
\end{abstract}

Keywords: Interleaved converter, PI control, Predictive current control, Average sliding control

\section{GIRISŞ (INTRODUCTION)}

Dağıtım sistemlerindeki harmonik akım kirlenmesi problemine ilgi son yıllarda önemli ölçüde artmıştır. Sinüzoidal akım dalga şeklinin bozulmasının negatif etkilerinden dolayı giriş akımının harmoniklerinin azaltılmasına ilişkin çalışmalar yapılmaktadır. Sistemlerin güç faktörünü düzeltmek için birçok arayüz devre çalışması yapılmaktadır. Genelde A.A. tarafının güç faktörünü düzeltmek için giriş akımının şeklini sinüzoidal forma aktif şekilde ayarlayan güç faktörü düzeltmeli dönüştürücüler kullanılmaktadır. 
Güç faktörü düzeltmeli dönüştürücüler, güç kaynağ çıkışını düzenleme ve aktif ve görünen güçte kabul edilebilen bir oran sağlamada güç elektroniğinin önemli bir çalışma ve araştırma alanıdır [1-6]. Yükselten, alçaltan, geriuçan, köprüsüz, sarmaşık yap1 gibi güç faktörü düzeltmeli dönüştürücülerde farklı topolojiler mevcuttur [7-11].

Yükselten güç faktörü önregülatörleri, basitlikleri (bir anahtar ve diyod), gerilim yükseltme karaktersitiği, verim ve performanslarından dolayı yaygın olarak kullanılmaktadır. Güç seviyesini arttırması bakımından uygun dönüştürücüler sıklıkla paralel veya seri şekilde bağlanarak kullanılırlar. Dönüştürücünün yüksek güçlerde tekli kullanılmasının en belirgin dezavantajı yüksek gerilim ve akım baskısına maruz kalmasıdır. $\mathrm{Bu}$ yüzden yüksek güç uygulamalarında klasik yükselten dönüştürücü türevi olan iki veya daha fazla yükselten dönüştürücünün sarmaşık yapıda bağlanması (anahtarlamalı dönüştürücülerin paralel bağlanması) akım dalgacığını azaltması bakımından önerilir [16]. $\mathrm{Bu}$ teknik aynı anahtarlama frekansında paralel çalışmada birçok hücrenin kontrol sinyalinin faz kaydırma işlemini ihtiva eder. Anahtarlama anları anahtarlama periyodunun eşit parçaları halinde sırayla faz kaydırılır. Bu işlem herhangi bir eleman üzerinde anahtarlama kayıplarını veya akım ve gerilim baskılarını arttırmaksızın tüm dönüştürücünün giriş akımı dalgacık genliğini düşürür ve etkin dalgacık frekansını arttırır. Düşük frekanslı harmoniklerin eliminasyonu filtreleme basamaklarının kayıplarının ve boyutunun azalmasını sağlar. Sarmaşık yapılı dönüştürücünün bu avantajları elektromanyetik girişimi azaltma, geçici tepkiyi arttırmadır [12-15].

Güç faktörü düzeltme yöntemi iki döngüyle gerçekleştirilir: bunlardan birisi birim güç faktörünü elde etmek için hızlı akım döngüsü, diğeri ise çıkış gerilimini düzenlemek için yavaş gerilim döngüsüdür. Oransal-İntegral akım kontrol yöntemi güç katsayısı düzeltme devrelerinde yaygın kullanılan tekniklerden bir tanesidir. Doğrultulmuş giriş akımı $\left|I_{\text {in }}\right|$, birim güç faktörünü elde etmek için doğrultulmuş giriş gerilimiyle orantılı olan referans akımı, $I_{\text {ref, takip }}$ etmeye zorlanır [9, 13, 14]. Güç dönüştürücülerde ortalama kayan kip kontrol yöntemi iki basamak içerir: birincisi ideal kayan yüzeyi elde etmek, ikincisi bu kayan yüzeyi çalışma frekansını sabitlemek veya üst sınırını belirlemek için modifiye etmektir. İdeal ortalama kayan kip kontrol algoritmaları genelde kolaydır fakat ikinci basamağın uygulamada sağlanmaya çalışılması işlemi zorlaştırmaktadır [20, 25]. Normalde geleneksel güç faktörü sağlayan dönüştürücülerde referans akımı elde etmek için çıkış gerilimi ölçülür ve referans gerilimle karşılaştırılır. Üretilen referans akım anahtarların görev oranını oluşturmak için ölçülen bobin akımıyla karşılaştırılır. $\mathrm{Bu}$ dönüştürücülerde çıkış gerilimi, bobin akımı, giriş gerilimi birim güç faktörünü sağlamak için ölçülmelidir. Öngörmeli akım kontrol yöntemini kullanarak bobin akımını ölçmeye gerek kalmaksızın birim güç faktörünü sağlamak mümkündür. $\mathrm{Bu}$ kontrol yöntemi birim güç faktörünü elde etmek için her bir anahtarlama anında bobin akımının tahminine dayanir [21].

Bu çalışmada, klasik tek anahtarlı DA-DA yükselten tip dönüştürücü yapısından türetilmiş iki anahtarlı sarmaşık yapılı yükselten devre (Şekil-2) mimarisi kullanılarak farklı kontrol yöntemlerinin analizi gerçekleştirilmiştir. Sarmaşık yapılı yükselten devre klasik yükselten devre ile aynı çalışma prensibine sahip olduğundan, devre analizi bu çalışma da aktarılmamıştır.

\section{UYGULANAN KONTROL YÖNTEMLERI (APPLIED CONTROL METHODS)}

Yıllardır güç faktörü düzeltme devrelerinin kontrolü için farklı akım kontrol yöntemleri uygulanmaktadır. Bir fazlı ve çok fazlı sarmaşık yapılı dönüştürücülerin kontrol yöntemleri temelde farklı değildir. Tek fark geri besleme döngüsü adedidir. N-fazlı dönüştürücü için N-adet geri besleme döngüsü mevcuttur. Sarmaşık yapılı devrede faz kaydırma işlemi sağlamak için ek devreye ihtiyaç duyulur. Bundan dolayı tüm kontrol teknikleri tek faza göre tasarlanıp görev oranı, $d_{2}$, sadece $180^{\circ}$ faz kaydırılmıştır. Bu bölümde giriş geriliminin temel bileşeniyle giriş akımını senkronize eden kontrol yöntemleri anlatılmıştır.

\subsection{Oransal-İntegral Kontrol Yöntemi (Proportional-Integral Control Method)}

Güç faktörü düzeltme kontrol yöntemlerinin temel amacı birim güç faktörünü sağlamak için dönüştürücünün direnç yükü gibi davranmasını sağlamaktır. Direnç yükü karakteristiğini elde etmek için giriş akımı giriş gerilimi değişimlerini takip etmelidir. Oransal-integral kontrolör bu işlem için yaygın kullanılan tekniklerden bir tanesidir. $\mathrm{Bu}$ yöntemde ortalama endüktör akımı alınır ve bu ortalama değer anahtarlama yapılarak kontrol edilir. Ortalama akım modlu oransal-integral kontrol [19, 22]'de detaylı analiz edilmiştir. (1) ve (2) no.lu eşitlikler oransal-integral kontrol yönteminin temel denklemlerini ifade etmektedir.

$$
\begin{aligned}
& I_{r e f}=\left[\left(V_{r e f}-V_{o}\right) \cdot V_{E A 1}\right] \cdot\left|V_{i n}\right| \\
& d_{1}=\left(I_{r e f}-\left|I_{i n}\right|\right) \cdot V_{E A 2} \\
& d_{2}=\left(I_{r e f}-\left|I_{i n}\right|\right) \cdot V_{E A 2} \cdot\left(180^{\circ}{ }_{\text {_ Phaseshift }}\right)
\end{aligned}
$$

burada $V_{E A I}$ : Oransal-integral kontrollü gerilim hata yükselteci, $V_{E A 2}$ : Oransal-integral kontrollü akım hata yükselteci $d_{1}$ ve $d_{2}$ sarmaşık yapılı dönüştürücünün sabit frekanslı rampa sinyali ile karşılaştırılarak üretilmiş görev oranlarını göstermektedir.

\subsection{Ortalama Kayan Kip Kontrol Yöntemi (Average Sliding Control Method)}

Ortalama kayan kip kontrol yöntemine göre tasarım yapıldığında güç dönüştürücüye ait bir anahtarlama 
denklemi elde edilir. İdeal durumda anahtarlama frekansı çok yüksek değerlere ulaşır buna rağmen daha yüksek frekans ideal kayan kipe daha iyi yaklaşmak demektir. Güç anahtarının karakteristiğinden dolayı bu yüksek frekansa bir üst limit konulmalıdır. Birçok uygulamada sabit anahtarlama frekansı arzu edilir, bazı durumlarda zorunludur. Bundan dolayı anahtarlama frekansını sınırlandırmak için çeşitli yöntemler mevcuttur. $\mathrm{Bu}$ yöntemin küçük sinyal analizi [23]'de detaylı analiz edilmiştir. (3) nolu eşitlik tek hücreli yükselten dönüştürücünün ortalama durum uzay modelinin temel denklemidir ve tasarım tek faza göre yapilmaktadir.

$\left|\dot{I}_{\text {in }}\right|=\left(\frac{1}{L}\right) \cdot\left|V_{\text {in }}\right|-\left(\frac{1-d}{L}\right) V_{o}$

$\left(\dot{I_{r e f}}-\left|\dot{I_{i n}}\right|\right)=-\lambda\left(I_{r e f}-\left|I_{i n}\right|\right)$

L. $\dot{I}_{r e f} \approx 0$ kabul edilip, $\left|\dot{I_{i n}}\right|$ eşitlik (4)'te yerine koyularak görev oranları eşitlik (5)' te türetilebilir.

$d_{1}=\frac{V_{o}-\left|V_{i n}\right|+\lambda L\left(I_{r e f}-\left|I_{\text {in }}\right|\right)}{V_{o}}$

$d_{2}=\frac{V_{o}-\left|V_{i n}\right|+\lambda L\left(I_{r e f}-\left|I_{i n}\right|\right)}{V_{o}} \cdot\left(180^{\circ}{ }_{-}\right.$Phaseshift $)$

burada, $\lambda=$ Kayan yüzey katsayısı $V_{E A l}$ : Oransalintegral kontrollü gerilim hata yükselteci, $d_{l}$ ve $d_{2}$ sarmaşık yapılı dönüştürücünün sabit frekanslı rampa sinyali ile karşılaştırılarak üretilmiş görev oranlarını göstermektedir. Güç dönüştürücülerde ortalama kayan kip kontrol yöntemi iki basamak içerir: birincisi ideal kayan yüzeyi elde etmek, ikincisi bu kayan yüzeyi çalışma frekansını sabitlemek veya üst sınırını belirlemek için modifiye etmektir. İdeal ortalama kayan kontrol algoritmaları genelde kolaydır fakat ikinci basamağın uygulamada sağlanmaya çalışılması işlemi zorlaştırmaktadır [24, 25].

\section{3. Öngörmeli Akım Kontrol Yöntemi (Predictive Current Control Method)}

Öngörmeli akım kontrol yöntemi son yıllarda güç dönüştürücülerde yaygın olarak kullanılmaya başlanmıştır. $\mathrm{Bu}$ yaklaşımda doğrultulmuş giriş gerilimi ve çıkış gerilimi $\left(\left|V_{i n}\right|, V_{0}\right)$ geri besleme sinyallerini, anahtarların $\left(M_{1}, M_{2}\right)$ kapı sinyalleri ise kontrolörünün çıkışını ifade eder. Çıkış gerilimi ve referans gerilim oransal-integral kontrolcüden geçirilerek $\left(V_{E A 1}\right)$ referans akımın büyüklüğü elde edilir. Üretilen referans akım, öngörmeli akım kontrol yöntemiyle bobin akımı ölçülmeksizin anahtarlama sinyallerinin oluşturulmasında kullanılır. Şekil 1 doğrultulmuş giriş hat gerilimini $\left|V_{\text {in }}(t)\right|$, doğrultulmuş giriş hat akımını $\left|I_{\text {in }}(t)\right|$, referans akımı $I_{\text {ref }}(t)$ ve çıkış gerilimini, $V_{0}(t)$ gösterir. Referans akım, giriş akım dalga şeklini düzeltmek ve birim güç faktörü elde etmek için giriş hat gerilimi ile orantılı olmalıdır. Zaman, $t=k T_{s}$ ve $k^{\text {th }}$ örnekleme anıdır, $T_{s}$ ise örnekleme periyodudur.

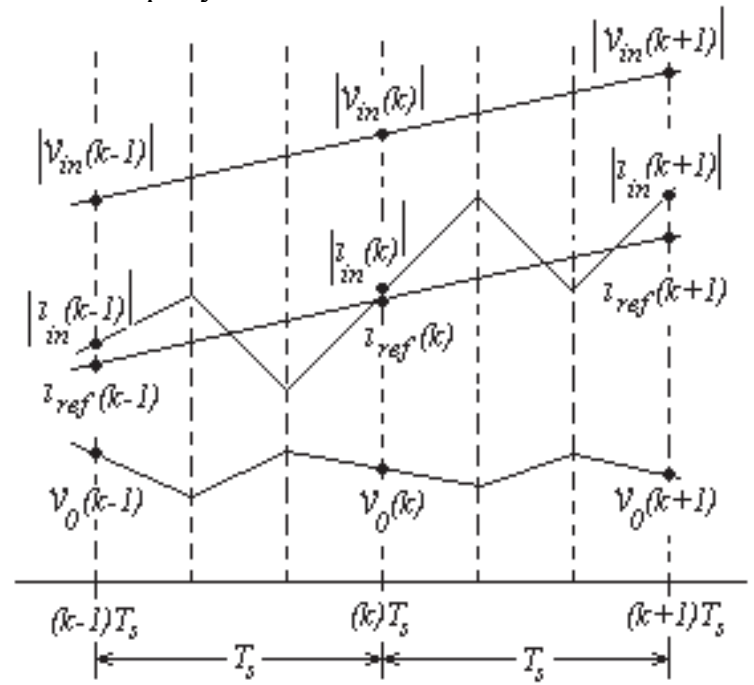

Şekil 1. $T_{s}$ için $(k-1)^{\text {th }}, k^{\text {th }}$ ve $(k+1)^{\text {th }}$ örnekleme anlarındaki akım ve gerilim dalga şekilleri (Current and voltage waveforms of $(k-1)^{\text {th }}, k^{\text {th }}$ and $(k+1)^{\text {th }}$ sampling intervals for $T_{s}$ )

Şekil 1'de görüldüğü gibi ortalama bobin akımı referans akımı her anahtarlama periyodunda takip etmelidir. $\left|I_{i n}(t)\right|$ akımı referans akımı, $I_{\text {ref }}(t)$, takip etmeye zorlanır. $V_{E A I}$ : Oransal-integral kontrollü gerilim hata yükseltecidir. Görev oranları ifadeleri kontrol yöntemine göre aşağıdaki şekilde türetilebilir:

$t_{k}<t<t_{k}+d_{k} T_{s} \& t_{k}+d_{k} T_{s}<t<t_{k+1}$ için,

$L \frac{d\left|I_{\text {in }}\right|}{d t}=d(k) T_{s}\left|V_{\text {in }}(k)\right|+\left(\left|V_{\text {in }}(k)\right|-\right.$

$\left.\left.V_{o}(k)\right)(1-d(k))\right) T_{s}$

$L \frac{d\left|I_{\text {in }}\right|}{d t}=d(k) T_{s}\left|V_{\text {in }}(k)\right|+\left|V_{\text {in }}(k)\right| T_{s}-$

$d(k) T_{S}\left|V_{\text {in }}(k)\right|-V_{o}(k) T_{S}+d(k) T_{S} V_{o}(k)$

$\left.V_{o}(k) T_{s}+d(k) T_{s} V_{o}(k)\right)$

$d(k)=\frac{V_{o}(k)-\left|V_{\text {in }}(k)\right|}{V_{o}(k)}+\frac{\left(\left|I_{\text {in }}(k+1)\right|-\left|I_{\text {in }}(k)\right|\right) L / T_{s}}{V_{o}(k)}$

burada, $\left|I_{\text {in }}(k+1)\right|=I_{\text {ref }}(k),\left|I_{\text {in }}(k+1)\right|=I_{\text {ref }}(k+1)$ ve $V_{o}(k)=V_{\text {ref }}$

$d_{1}(k)=\frac{V_{r e f}-\left|V_{i n}(k)\right|}{V_{r e f}}+\frac{\left(I_{r e f}(k+1)-I_{r e f}(k)\right) L / T_{s}}{V_{r e f}}$

$d_{2}(k)=\frac{V_{r e f}-\left|V_{\text {in }}(k)\right|}{V_{\text {ref }}}+$

$\frac{\left(I_{\text {ref }}(k+1)-I_{\text {ref }}(k)\right) L / T_{s}}{V_{\text {ref }}} \cdot\left(180^{\circ}{ }_{-}\right.$Phaseshift $)$

Öngörmeli akım kontrol yöntemi güç dönüştürücüleri için birçok avantaj sunar: kolay anlaşılır, birçok 
sisteme uygulanabilir ve diğer değişik durumlar için kolay uygulanabilir bir kontrolördür. $\mathrm{Bu}$ yöntem klasik kontrol yöntemiyle karşılaştırıldığında çok sayıda hesaplama içerir fakat günümüzde bu uygulamayı gerçekleştirecek hızlı mikroişlemciler mevcuttur [21].

\section{BENZETIM VE DENEYSEL ÇALIŞMA SONUÇLARI (SIMULATION AND EXPERIMENTAL RESULTS)}

Benzetim çalışmaları MATLAB/Simulink programı kullanılarak gerçekleştirilmiştir. Benzetim çalışmalarında seçilen parametreler Tablo 1'de gösterilmiştir. Sarmaşık yapılı dönüştürücü ise Şekil 2'de gösterilmiştir.
Tablo 1. Benzetimde seçilen parametreler (Selected simulation parameters)

\begin{tabular}{llr}
\hline \hline Çıkış gücü & $P_{0}$ & $1[\mathrm{~kW}]$ \\
\hline Çıkış gerilimi & $V_{0}$ & $400[\mathrm{~V}]$ \\
Giriş gerilimi & $V_{\text {in }}, f_{\text {line }}$ & $220\left[\mathrm{~V}_{\mathrm{rms}}, 50 \mathrm{~Hz}\right]$ \\
Anahtarlama & $f_{s}$ & $50[\mathrm{kHz}]$ \\
frekansı & & $6,8[\mathrm{mH}]$ \\
Bobin değerleri & $L_{1}=L_{2}$ & $500[\mu \mathrm{F}]$ \\
Çıkış kapasitörü & $C_{0}$ & \\
\hline \hline
\end{tabular}

Gerilim kontrolcüler tüm kontrol yöntemlerine göre Şekil 3'te gösterildiği gibi aynı yapıdadır. Şekil 4-6 sirasiyla oransal-integral, ortalama kayan kip ve öngörmeli akım kontrol yöntemlerini göstermektedir. Oransal-integral kontrolörde, $K_{p}$ ve $K_{i}$ ZieglerNichols yöntemine göre seçilir.

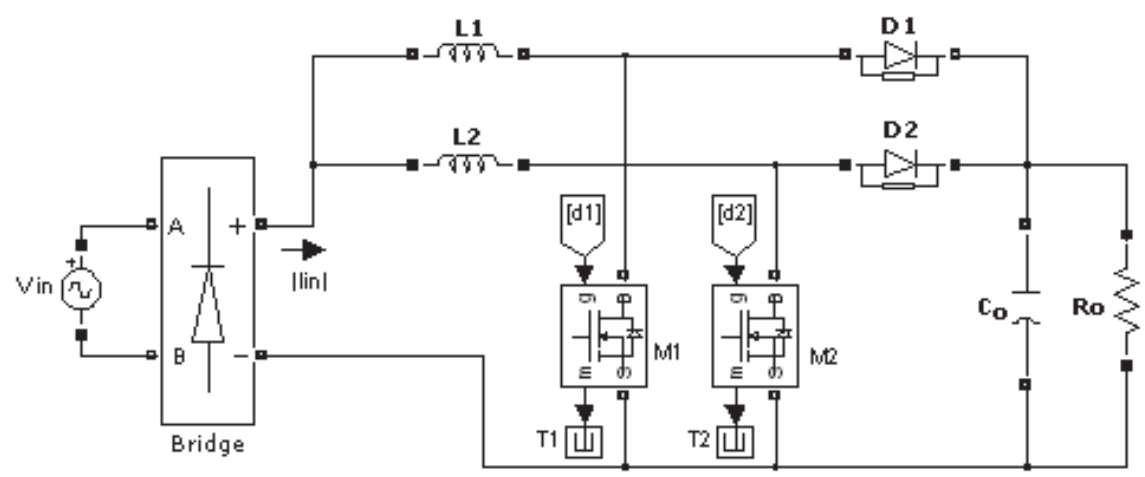

Şekil 2. Sarmaşık yapılı dönüştürücü topolojisi (The interleaved converter topology)

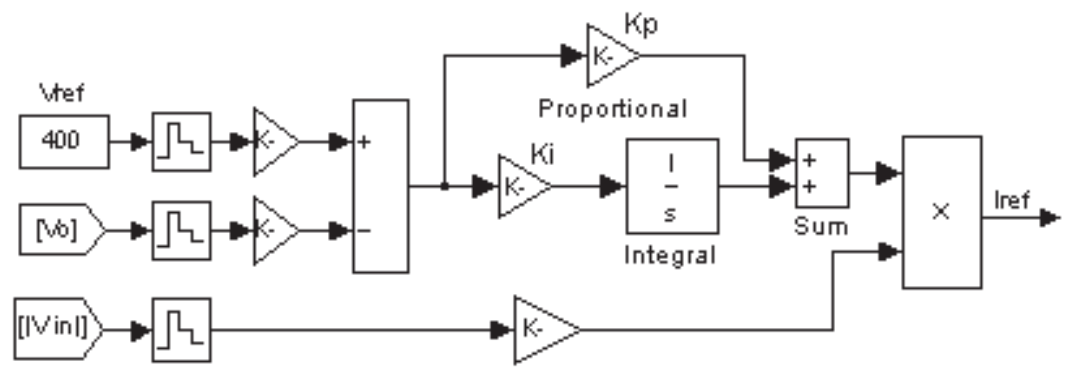

Şekil 3. Gerilim kontrolcü bloğu (Voltage controller block set)

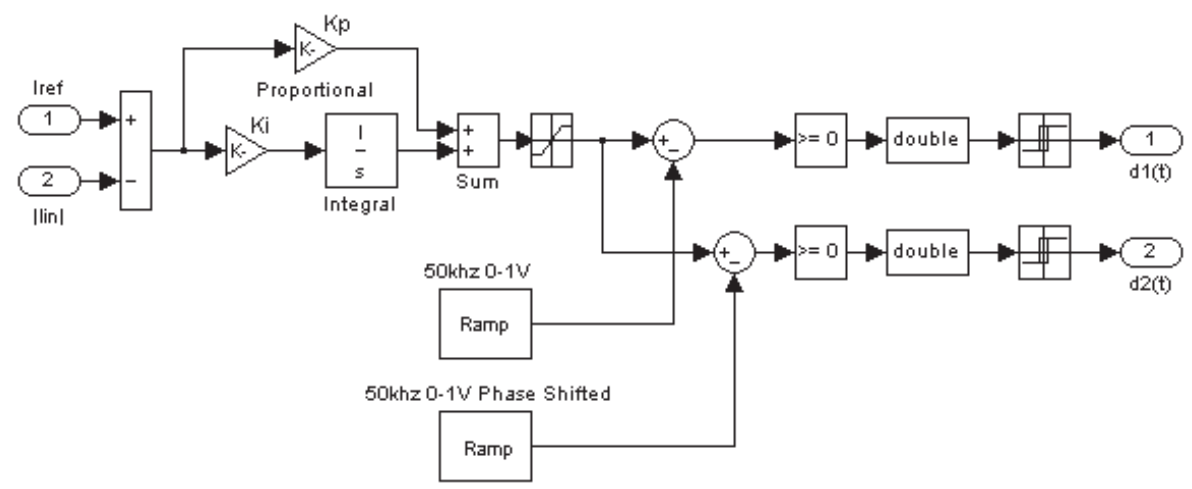

Sekil 4. Oransal-integral akım kontrolcüsü (Proportional-integral current controller) 


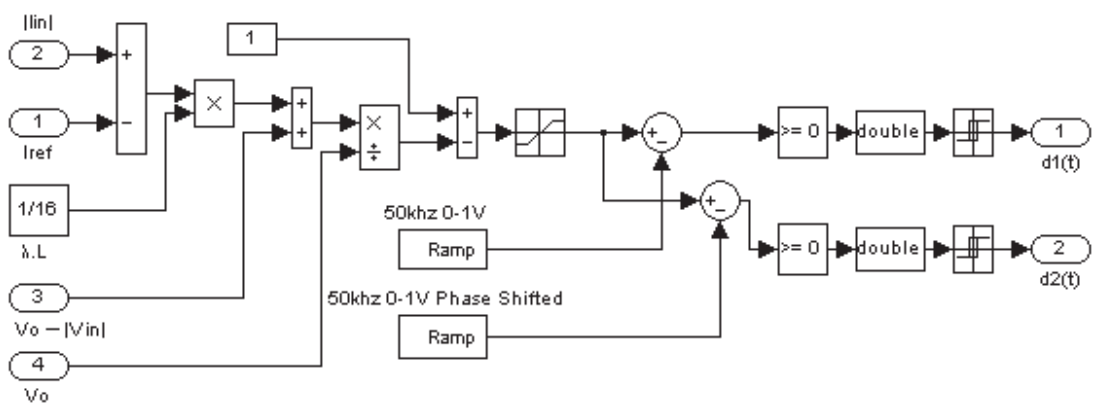

Şekil 5. Ortalama kayan kip akım kontrolcüsü (Average sliding current controller)

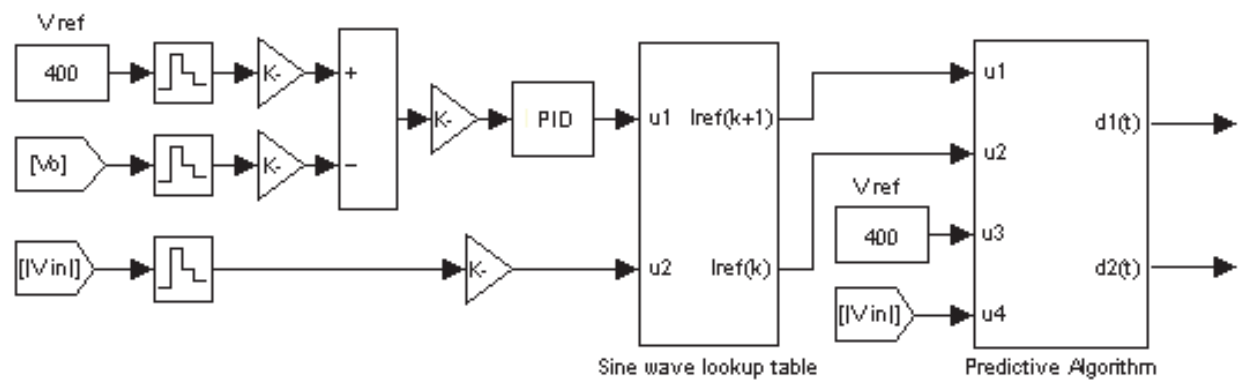

Şekil 6. Öngörmeli akım kontrolcüsü (Predictive current controller)

Şekil 7 oransal-integral yöntemine göre giriş hat akımı, giriş ve çıkış gerilimi değişiminin benzetim sonuçlarını göstermektedir. $\mathrm{Bu}$ durum için giriş gerilimi $220 \mathrm{~V}_{\text {rms }}$, çıkış gücü $1 \mathrm{~kW}$, giriş gerilim frekansı 50 Hz'dir. Şekil 7(a), (b)'de dönüştürücü gücü $1 \mathrm{~kW}$ 'dan $500 \mathrm{~W}$ 'a ve $500 \mathrm{~W}^{\prime}$ tan $1 \mathrm{~kW}$ 'a değiştirilerek sonuçlar gösterilmiştir. Güç katsayısı ve toplam harmonik bozulma $1 \mathrm{~kW} / 500 \mathrm{~W}$ değerlerine göre sirasıyla $0,99 / 0,99$ ve $7,1 \% / 8,3 \%$ ' dir.

Şekil 8 ortalama kayan kip yöntemine göre giriş hat akımı, giriş ve çıkış gerilimi değişiminin benzetim sonuçlarını göstermektedir. Giriş gerilimi $220 \mathrm{~V}_{\mathrm{rms}}$, çıkış gücü $1 \mathrm{~kW}$, giriş gerilim frekansı $50 \mathrm{~Hz}$ iken Şekil 8(a), (b)'de dönüştürücü gücü $1 \mathrm{~kW}$ 'dan 500 W'a ve 500W'tan $1 \mathrm{~kW}$ 'a değiştirilerek sonuçlar gösterilmiştir. Güç katsayısı ve toplam harmonik bozulma $1 \mathrm{~kW} / 500 \mathrm{~W}$ değerlerine göre sirasiyla $0,99 / 0,99$ ve $6,0 \% / 6,2 \%$ 'dir.

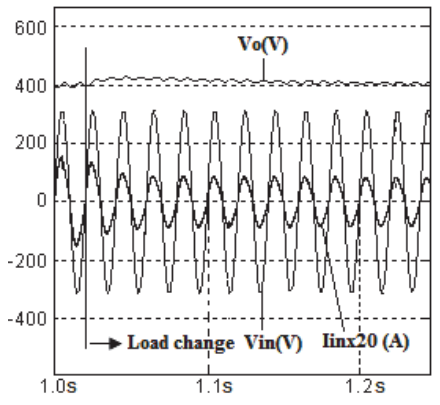

(a)
Şekil 9 öngörmeli akım yöntemine göre giriş hat akımı, giriş ve çıkış gerilimi değişiminin benzetim sonuçlarını göstermektedir. $\mathrm{Bu}$ durum için giriş gerilimi $220 \mathrm{~V}_{\mathrm{rms}}$, çıkış gücü $1 \mathrm{~kW}$, giriş gerilim frekansı $50 \mathrm{~Hz}$ 'dir. Şekil 9(a), (b)'de dönüştürücü gücü $1 \mathrm{~kW}$ 'dan 500 W'a ve 500 W'tan $1 \mathrm{~kW}$ 'a değiştirilerek sonuçlar gösterilmiştir. Güç katsayısı ve toplam harmonik bozulma $1 \mathrm{~kW} / 500 \mathrm{~W}$ değerlerine göre sirasiyla $0,99 / 0,99$ ve $6,2 \% / 15,8 \%$ 'dir.

Tüm kontrol yöntemleri için sarmaşık yapılı tek fazlı doğrultucu anahtarlama frekansı $50 \mathrm{kHz}$ alınmıştır. Tüm kontrol tekniklerine göre sonuçlar Tablo 2'de sunulmuştur

Şekil 7. Benzetim sonucu; giriş, çıkış gerilimi ve hat akımı değişimi: (a) 1kW'dan 500 W'a, (b) 500W'tan 1

$\mathrm{kW}$ 'a (Input line, output voltage and the line current changes: (a) from $1 \mathrm{~kW}$ to $500 \mathrm{~W}$. (b) from 500 to $1 \mathrm{~kW}$ ) 

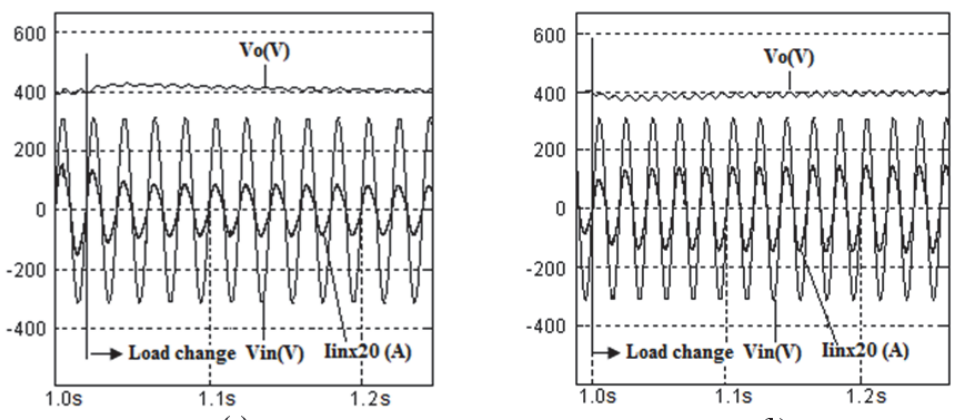

(b)

Şekil 8. Benzetim sonucu; giriş, çıkış gerilimi ve hat akımı değişimi: (a) 1kW'dan 500 W'a, (b) 500W'tan 1 kW'a (Input line, output voltage and the line current changes: (a) from $1 \mathrm{~kW}$ to $500 \mathrm{~W}$, (b) from 500 to $1 \mathrm{~kW}$ )

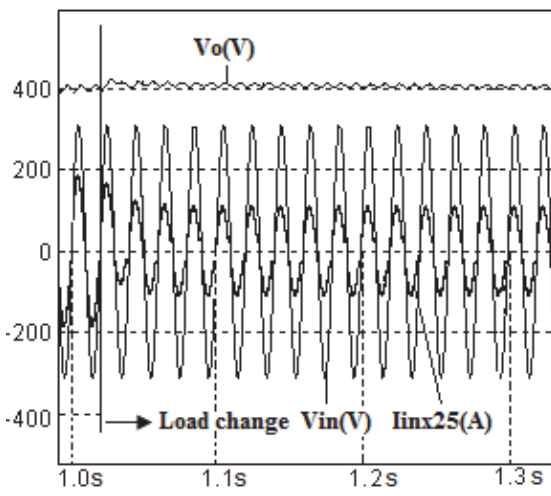

(a)

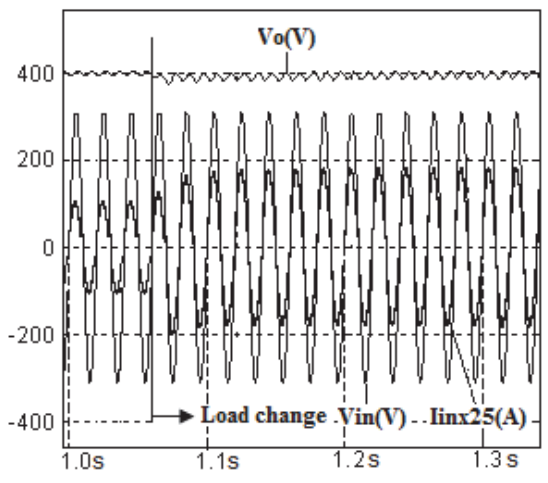

(b)

Şekil 9. Benzetim sonucu; giriş, çıkış gerilimi ve hat akımı değişimi: (a) 1kW'dan 500 W'a, (b) 500W'tan 1 $\mathrm{kW}$ 'a (Input line, output voltage and the line current changes: (a) from $1 \mathrm{~kW}$ to $500 \mathrm{~W}$, (b) from 500 to $1 \mathrm{~kW}$ )

Tablo 2. Detaylı benzetim sonuçları (Detailed simulation results)

\begin{tabular}{|c|c|c|c|c|c|c|c|}
\hline Benzetim No & 1 & 2 & 3 & 4 & 5 & 6 & 7 \\
\hline \multicolumn{8}{|l|}{ Parametreler } \\
\hline$G \ddot{u c ̧}(k W)$ & 1 & 1 & 1 & 1 & 0,5 & 1 & 1 \\
\hline$L_{1}=L_{2}(m H)$ & 6,8 & 6,8 & 6,8 & 5,0 & 6,8 & 0,8 & 6,8 \\
\hline$C_{o}(\mu F)$ & 500 & 500 & 500 & 500 & 500 & 500 & 250 \\
\hline$V_{\text {in }}(V r m s)$ & 220 & 110 & 250 & 220 & 220 & 220 & 220 \\
\hline \multicolumn{8}{|c|}{ Oransal-integral yöntemi } \\
\hline$P F$ & 0,99 & 0,99 & 0,99 & 0,99 & 0,99 & 0,99 & 0,99 \\
\hline$T H D(\%)$ & 7,1 & 2,7 & 9,1 & 7,3 & 8,3 & 35 & 13,3 \\
\hline \multicolumn{8}{|c|}{ Ortalama kayan kip yöntemi } \\
\hline$P F$ & 0,99 & 1 & 0,99 & 0,99 & 0,99 & 0,99 & 0,99 \\
\hline$T H D(\%)$ & 6,0 & 1,7 & 8,6 & 6,5 & 6,2 & 10 & 13,1 \\
\hline \multicolumn{8}{|c|}{ Öngörmeli akım kontrol yöntemi } \\
\hline$P F$ & 0,99 & 1 & 0,99 & 0,99 & 0,99 & 0,93 & 0,99 \\
\hline THD (\%) & 6,2 & 8,9 & 6,8 & 15 & 15,8 & 43 & 10,0 \\
\hline
\end{tabular}




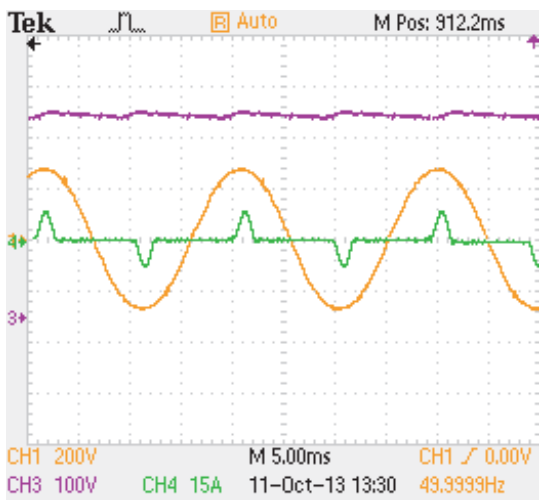

a)

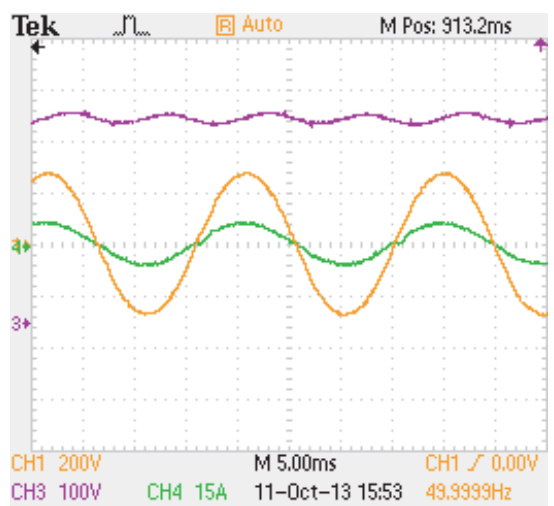

b)

Şekil 10. Deneysel çalışma; sarmaşık yapılı tek-fazlı doğrultucunun çıkış gerilimi, giriş akımı ve gerilimi dalga şekilleri: a) Kontrolsüz çalıștırma $(\% \mathrm{THD}=\% 65)$, b) Birim güç katsayılı kontrollü çalıștırma

$(\% \mathrm{THD}=\% 7.5)$ (Input line, output voltage and the line current changes: (a) without controller, (b) with control)

Sarmaşık yapılı tek-fazlı doğrultucu için deneysel çalışmalar yapılmış ve sürekli iletim durumunda PI kontrol yöntemi kullanılarak elde edilen sonuçların analizi yapılmıştır. Deneysel çalışmalarda doğrultucunun kontrolü DSP TMS320F2812 mikroişlemcisi kullanılarak gerçekleştirilmiştir. Şekil 10.'da sarmaşık yapılı tek-fazlı doğrultucunun $1 \mathrm{~kW}$ çıkış gücünde çalıştırılarak çıkış gerilimi, giriş gerilimi ve akımı dalga şekilleri verilmiştir. Şekil 10 a'dan anlaşılacağı üzere devre kontrolsüz olarak çalıştırıldığında giriş akımı dalga şekli bozuk ve THD değeri \%65 seviyesindedir. Şekil 10-b'de ise devre birim güç faktörü için kontrollü olarak çalıştırılmaktadır. Dalga şekillerinden anlaşılacağ 1 üzere giriş akımı giriş gerilimini takip ederek güç faktörü \%99 seviyesinde sabit tutulmaktadır. Giriş akım sinyalinin THD değerine bakıldığında ise değerin $\% 7,5$ civarında olduğu ve eş değer benzetim çalışma sonucu ile yaklaşık örtüştüğü gözlenmiștir.

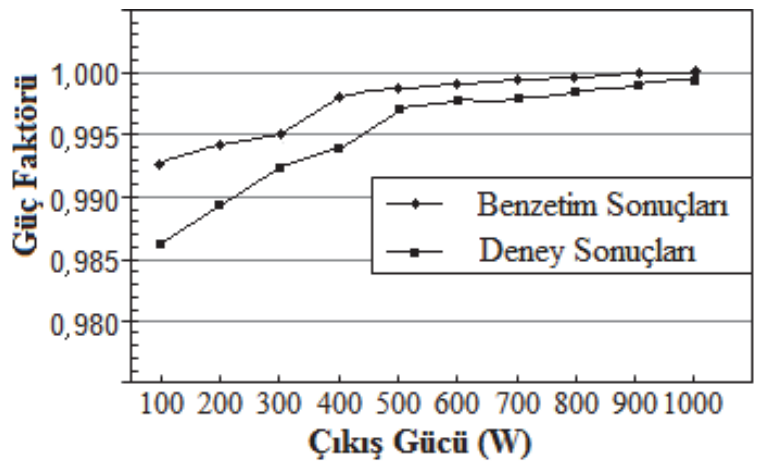

Şekil 11. $220 \mathrm{~V}_{\mathrm{rms}}$ giriş geriliminde çıkış gücü değerlerine karşı güç faktörü (Input power factor versus output power at $220 \mathrm{~V}_{\text {rms }}$ input voltage)

Devre güç katsayısı düzeltimi için kontrollü olarak çalıştırıldığında giriş akımı harmoniklerini ciddi bir şekilde azalttığı ve uluslar arası harmonik standartlarına uygun hale getirdiği gözlenmiştir. Sarmaşık yapılı tek-fazlı doğrultucunun farklı çıkış güç değerlerine karşın PI kontrolü ile elde edilen benzetim çalışmaları ve deneysel sonuçlarda elde edilen güç faktörü sonuçları Şekil 11 'de verilmiştir. Belirli bir güç değerinden sonra devrenin deneysel çalışma ve benzetim çalışma sonuçlarının büyük oranda örtüştüğü gözlenmiştir.

\section{SONUÇ (CONCLUSION)}

Oransal-integral tabanlı ortalama akım kontrollü sarmaşık yapılı yükselten devre tasarlanarak hem benzetim hem de deneysel çalışmaları yapılmış ve benzetim sonuçları ile deneysel çalışma sonuçlarının bir biri ile örtüştüğü gözlenmiştir. Bu nedenle, bu çalışmada, oransal-integral, ortalama kayan kip ve öngörmeli kontrol yöntemleri sarmaşık yapılı yükselten tip güç katsayısı düzeltici devreye uygulanıp Matlab/Simulink programında benzetimleri gerçekleştirilmiştir. Elde edilen sonuçların detaylı analizleri yapılmıştır.

Kullanılan tüm kontrol tekniklerinde sabit anahtarlama frekansı kullanılarak pratik çalıșmalar için uygulanabilirliği sağlanmıştır. Öngörmeli kontrol yönteminde işlemlerin bobin akımı ölçülmeden yapılması diğer iki kontrol yöntemine göre daha avantajlı olduğu göstermiştir. Bunun için öngörmeli akım kip kontrol yönteminde gerilim kontrolcüsü aynı zamanda endüktör akımını da kontrol etmektedir. Tasarlanan tüm kontrol yöntemlerinin çıkıș yükü değişimlerine karşı dayanıklı bir yapı sergilediği gözlemlenmiştir.

Endüktör değeri azaltıldığında oransal-integral kontrol ve öngörmeli akım kip kontrolde kullanılan kontrolcülerin katsayı ayarlamalarının tekrar yapılması gerektiği ortalama kayan kip yönteminin ise herhangi bir değişiklik yapılmadan performansını koruduğu gözlemlenmiştir.

$\mathrm{Bu}$ çalışma ile öngörülen kontrol teknikleri pratik çalışmalara da uyarlanarak elde edilen sonuçların analizi yapılabilir. 


\section{TEŞEKKÜR (ACKNOWLEDGMENT)}

Bu çalışma Türkiye Bilimsel ve Teknolojik Araştırma Kurumu (TÜBİTAK) tarafindan desteklenen 108E081 ve 111E171 No'lu projelerden üretilmiştir. Katkılarından dolayı TÜBİTAK'a teşekkürü bir borç biliriz.

\section{KAYNAKLAR (REFERENCES)}

1. Wolfle, W.H. ve Hurley, W.G., "Quasi-active power factor correction with a variable inductive filter, theory, design and practice", Power Elec. IEEE Transactions, Cilt 18, 248-255, 2003.

2. Huai, W., Batarseh, I., Guangyong, Z., ve Kornetzky P., "A single-switch AC-DC converter with power factor correction", Power Elec., IEEE Transactions, Cilt 15, No 3, 421-430, 2000.

3. Mather, B.A. ve Maksimović, D.,"A Simple Digital Power-Factor Correction Rectifier Controller", Power Electronics IEEE Transactions, Cilt 26, No 1, 9-19, 2001.

4. Tsai-Fu, W., Chien-Chih, C., Chih-Lung, S., ve Cheng-Nan, W., "Analysis, design, and practical considerations for $500 \mathrm{~W}$ power factor correctors", Aerospace and Electronic Systems IEEE Transactions, Cilt 39, No 3, 961-975, 2003.

5. GENC, N. ve Iskender, I., "An Improved Soft Switched PWM Interleaved Boost AC-DC Converter", Energy Conversion and Management., Cilt 52, No. 1, 403-413, 2011

6. Karaarslan, A., ve Iskender, I., "Pure sinusoidal input voltage based bridgeless PFC converter using TMS320F2812 digital signal processor", Electrical and Electronics Engineering, ELECO'09 International Conf., 234-238, 2009.

7. Qiao, C., ve Smedley, K.M.,'A topology survey of single-stage power factor corrector with a boost type input-current-shaper", Power Electronics IEEE Transactions, Cilt 16, No 3, 360-368, 2001.

8. Po-Wa, L., Yim-Shu, L., Cheng, D.K.W., ve XiuCheng, L., "Steady-state analysis of an interleaved converter with coupled inductors", Industrial Electronics IEEE Transactions, Cilt 47, No 4, 787-795, 2000.

9. Yao-Ching, H., Te-Chin, H., ve Hau-Chen, Y., "An Interleaved Boost Converter with Zero-Voltage Transition", Power Electronics IEEE Transactions, Cilt 24, No 4, 973-978, 2009.

10. Alonso, J.M., Dalla Costa, M.A., ve Ordiz, C., "Integrated Buck-Flyback Converter as a HighPower-Factor Off-Line Power Supply", Industrial Electronics IEEE Transactions, Cilt 55, No 3, 1090-1100, 2008.

11. Jun, Z., Lu, D.D.-C., ve Ting, S., "Flyback-Based Single-Stage Power-Factor-Correction Scheme with Time-Multiplexing Control", Industrial Electronics IEEE Transactions, Cilt 57, No 3,1041-1049, 2010.

12. Yao, G., Chen, A., ve He, X., "Soft Switching Circuit for Interleaved Boost Converters", IEEE Transactions on Power Electronics, Cilt 22, No 1, 80-86, 2007.
13. Hsieh, Y.-C., Hsueh T.-C., ve Yen, H.-C., "An Interleaved Boost Converter With Zero-Voltage Transition", IEEE Transactions on Power Electronics, Cilt 24, No 4, 973-978, 2009.

14. Yungtae, J.,ve Jovanovic M.M., "Interleaved Boost Converter with Intrinsic Voltage-Doubler Characteristic for Universal-Line PFC Front End", IEEE Power Electronics, Cilt 22, No 4, 13941401, 2007.

15. Rezvanyvardom, M., ve All E., "A New Interleaved ZCS PWM Boost Converter", IEEE International Conference on Power and Energy, Cilt 1, 45-50, 2010.

16. IEC Standard IEC 61000-3-2,"Electromagnetic Compatibility (EMC), Limits for Harmonic Current Emissions (Equipment input current $\leq 16 \mathrm{~A}$ per phase)", 2009.

17. GENC, N., ve Iskender, I., "DSP-based Current Sharing of Average Current Controlled Two-Cell Interleaved Boost PFC Converter", IET Power Electronics, Cilt 4, No. 9, 1015-1022, 2011.

18. Pinheiro, J. R., Gründling, H. A., Vidor, D. L.R., ve Baggio, J.E., "Control Strategy of an Interleaved Boost Power Factor Correction Converter", IEEE Annual Power Electronics Specialists Conf., Cilt $1,137-142,1999$.

19. Karaarslan, A., ve Iskender, I., "Güç Katsayısı Düzeltim Devrelerinde Ortalama Akım Kontrol Tekniği ve Sayısal Sinyal İşlemcisi Kullanılarak Yeni bir Yöntemin Uygulanması", Journal of the Faculty of Engineering and Architecture of Gazi University, Cilt 26, No 1, 193-203, 2011.

20. Navarro-López, E.M., Cortés, D., ve Castro, C., "Design of practical sliding-mode controllers with constant switching frequency for power converters", Electric Power Systems Research, Cilt 79, 796-802, 2009.

21. Zhang, W., Feng, G., Liu, Y.-F., ve Wu, B., "A digital power factor correction (PFC) control strategy optimized for DSP”, IEEE Trans. Power Electronics, Cilt 19, No 6, 1474-1485, 2004.

22. Sun, C., ve Lehman, B., "Discussions on Control Loop Design in Average Current Mode Control", IEEE Industry Application Conf., Cilt 4, 24112417, 2000.

23. Mattavelli, P., Rossetto, L., ve Spiazzi, G., "SmallSignal Analysis of DC-DC Converters with Sliding Mode Control", IEEE Trans. Power Electronics, Cilt 12, No 1, 96-102, 1997.

24. Navarro-López, E.M., Cortés, D., ve Castro, C., "Design of practical sliding-mode controllers with constant switching frequency for power converters", Electric Power Systems Research, Cilt 79, 796-802, 2009.

25. A. Karaarslan, I. Iskender, "Average Sliding Control Method Applied on PFC Converter for Decreasing Input Current THD using Digital Signal Processor", IET Power Electronics, Cilt 5, No. 5, 617-626, 2012. 